\title{
Image Enhancement using Hybrid Fuzzy Inference System (IEHFS)
}

\author{
Kumud Saxena \\ Assistant Professor \\ FMCA,RBS College, Agra
}

\author{
Avinash Pokhriyal \\ Reader \\ FMCA, RBS College, Agra
}

\author{
Sushma Lehri, PhD. \\ Director \\ IET, Dr B R Ambedkar \\ University, Agra
}

\begin{abstract}
Image enhancement is a primary need for the recognition of different biometrics in biometric-based identification systems. The recognition-rate of a biometric system depends heavily upon the quality of the input biometric given to the system. In this paper, a novel hybrid Fuzzy model (IEHFS) is proposed to improve the visual quality of iris images. The experimental results based on calculating PSNR values show that this hybrid model enhances the noisy biometric images better than the conventional filters like median filter and Wiener filter.
\end{abstract}

\section{General Terms}

Image Enhancement, Iris enhancement, Fuzzy Inference System.

\section{Keywords}

IEHFS, Wiener filter, Median filter, Top hat and bottom hat, PSNR, SSIM

\section{INTRODUCTION}

Biometric identification has taken the highest priority in identification techniques as it is secure, easily accessible and no need to remember it. Iris is one of the best ways among other biometric identification as it has less chances of getting changed, damaged, less effect of age, and no theft. But the image can acquire different types of noises due to incorrect image acquisition and storing [1]. There can be many types of noises encountered due to several reasons like the impulse noise (or salt and pepper noise) caused by some sudden changes or sharp disturbances in the image signal; due to which white or black or both pixels scatter or appear over the image. Such types of noise can be removed by using spatial filtering techniques like adaptive or median filtering.

Adaptive filtering produces good results by preserving edges and other high-frequency parts of an image. Hence wiener filter is applied to enhance the noisy image.

Median filter achieves higher performance when the image contains large domain matrices and do not contain any zerovalued elements. Median filters have been applied heavily to image processing problems [2].

Both the filters are applied on biometric images containing salt \& pepper noise. Section II and III show the implementation of Wiener and median filters on Iris images.

Much work has been done to enhance the images. Softcomputing has recently been applied to image denoising process. Among all, Fuzzy technique is a promising technique for image enhancement [5]. It gives better results as compared to median and wiener filter[6]. These fuzzy filters, including FIR-filter [7], the weighted fuzzy mean filter [8], and the iterative fuzzy control based filter [9], do not produce very clear results for Gaussian noise. In this paper iris image is enhanced using hybrid fuzzy model based on the Mamdani model. Section IV discusses the working of the system. In section $\mathrm{V}$, the experimental results and comparison among the three techniques are shown and conclusions are drawn in the last section.

Digital images can acquire variety of noise. It is the result of errors in the image acquisition process and it changes the real intensity of the pixel. An image can have noise due to various reasons; MATLAB toolbox provides a function by which these noises can be added into the image. The 'imnoise' function will be used here for finding the best denoising method.

\section{WIENER FILTER}

Wiener filtering [1] is an effective linear image restoration approach. It is based on estimating the statistics from a local neighbourhood of a pixel. The task is to find the estimate of the "best" image from the degraded image. Image processing toolbox of Matlab offers functions like wiener2 which uses a pixel-wise adaptive Wiener method using neighborhoods of size $m \times n$ to estimate the local image mean and standard deviation. Where the variance is large, it performs little smoothing. Where the variance is small, wiener2 performs more smoothing. Wiener works on gray scale so the image is first converted to grayscale. Then wiener is applied. The Figure 1 shows the process.

The algorithm applied through MATLAB on Iris image to see the results

1. Read the image RGB = imread('002_1_1.bmp'); imshow('001_1_1.bmp')

2. Change the image to grayscale $\mathrm{I}=\operatorname{rgb2gray}(\mathrm{RGB})$; imshow(I)

3. Add noise to the image $\mathrm{J}=$ imnoise(I,'salt \& pepper',0.02); imshow(J)

4. Remove noise by applying wiener $\mathrm{K}=$ wiener2(J,[3 3$]$ ); figure, imshow(K) 


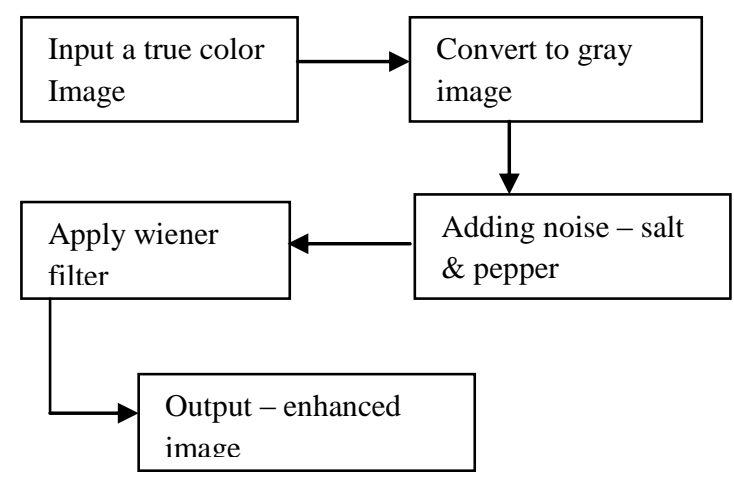

Fig 1: Applying wiener filter

\section{MEDIAN FILTER}

Averaging filter, in which each output pixel is set to an average of the pixel values in the neighborhood of the corresponding input pixel. Median filtering similar to it, the value of output pixel is determined by the median of the neighborhood. Median filtering is a nonlinear operation often used in image processing to reduce "salt and pepper" noise and preserve edges[10]. Here median filter is applied on Iris Image to remove this type of noise. Figure 2 shows the applied process.

The algorithm applied through MATLAB on the Iris image

1. Read the image

I = imread('002_1_1.bmp');

imshow(I)

2. Add noise to the image

$\mathrm{J}=$ imnoise (I,'salt \& pepper',0.02);

imshow $(\mathrm{J})$

3. Remove noise by applying median

$\mathrm{K}=$ medfilt2(J,[3 3$]$ );

figure, imshow(K)

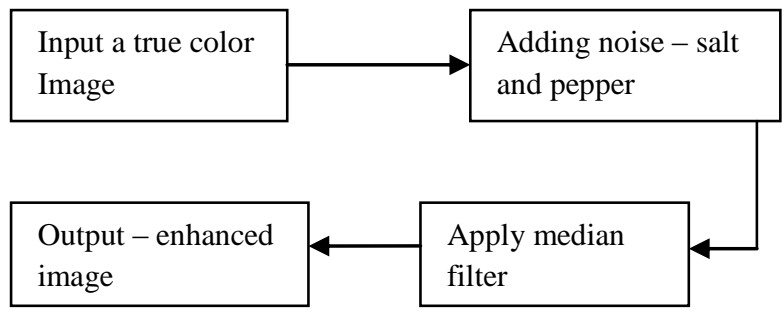

Fig 2: Applying median filter

However, when the noise level is over 50\%, some details and edges of the original image are smeared by the filter [11]. Different approches of the median filter have been proposed, e.g. the adaptive median filter [12], the multi-state median filter [13], or the median filter based on homogeneity information [14] and [15].

\section{IMAGE ENHANCEMENT USING HYBRID FUZZY INFERENCE SYSTEM (IEHFS)}

Fuzzy inference maps the given input to the desired output using fuzzy logic by using membership functions, logical operations and IF-THEN rules. Fuzzy system can be classified in two types, Mamdani-type and Sugeno-type. The first one includes linguistic models based on collections of IF-THEN rules, whose antecedents and consequents utilize fuzzy sets. The Mamdani model [16] follows it. The second category, based on Takagi-Sugeno (TS) model systems [17], uses a rule structure that has fuzzy antecedent and functional consequent parts.

In this paper, hybrid Fuzzy inference system is applied by taking a movable window over the image of $5 \times 5$ size, where the pixels around the center pixel are named according to the direction to identify them. Their gray intensity is measured and a comparison is made in one direction to the other adjacent pixels to find their intensity level and then a new value is assigned according to the fuzzy rules and function. The output image is then passed through top and bottom hat filters to get a much enhanced image. The Iris image enhancement using the proposed IEHFS process is shown in figure 3 . The proposed algorithm to enhance the Iris image has the following steps:

1. Read the image.

2. If image is true colour, convert it to gray image

3. The gray image $\mathrm{I}$ is considered to be of size $\mathrm{mx} \mathrm{n}$ where $I=\{I(i, j) \in\{1,2, \ldots . .255\}\}$ and $i=1,2 \ldots \ldots . m$ and $\mathrm{j}=1,2, \ldots \ldots . . \mathrm{n}$.

4. A movable window $\mathrm{W}$ of size $5 \times 5$ is taken as shown in figure 4.

5. The fuzzification of the image is done, as shown by the matrix in figure 5 .

6. Fuzzy rules are applied to enhance the image.

7. Top and bottom hat filters are applied on enhanced image.

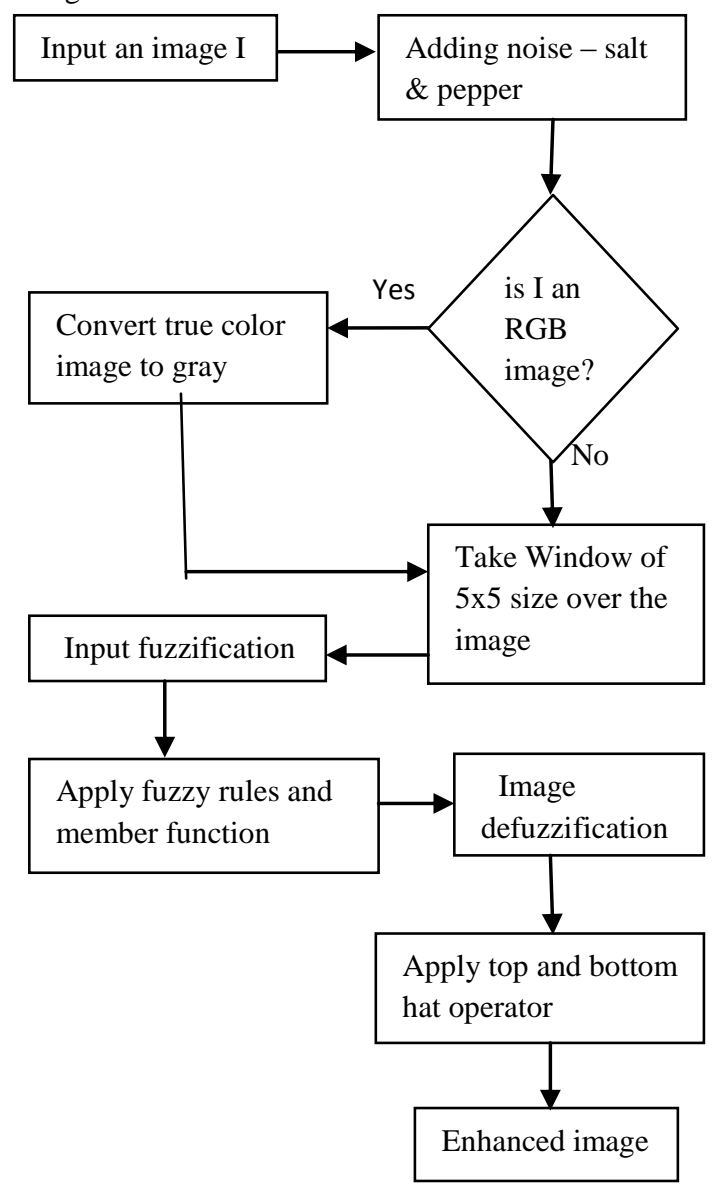

Fig 3: Applying IEHFS 


\begin{tabular}{|c|c|c|c|c|}
\hline NW1 & NNW & N1 & NNE & NE1 \\
\hline NWW & NW & N & NE & NEE \\
\hline W1 & W & P(i.j) & E & E1 \\
\hline SWW & SW & S & SE & SEE \\
Pixel \\
\hline SW1 & SSW & S1 & SSE & SE1 \\
\hline
\end{tabular}

Fig 4 :Dimensions of movable window $W$

$\left(\begin{array}{lllll}i-2, j-2 & i-2, j-1 & i-2, j & i-2, j+1 & i-2, j+2 \\ i-1, j-2 & i-1, j-1 & i-1, j & i-1, j+1 & i-1, j+2 \\ i, j-2 & i, j-1 & i, j & i, j+1 & i, j+2 \\ i+1, j-2 & i+1, j-1 & i+1, j & i+1, j+1 & i+1, j+2 \\ i+2, j-2 & i+2, j-1 & i+2, j & i+2, j+1 & i+2, j+2\end{array}\right)$

Fig 5 : Matrix of image input fuzzification

In the filtering Window $\mathrm{W}$ each pixel corresponds to the adjacent pixel by the given direction. The basic assumption in this technique is that the new pixel must be taken from the Window W. After fuzzification, the fuzzy rules applied are

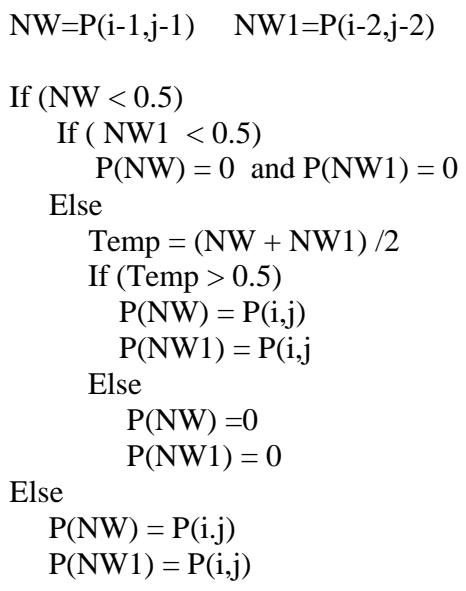

In this way, each pixel under 5 x 5 window is being checked with the center pixel intensity. If the intensity is less than $50 \%$, then it is assigned the value 0 else it is equal to the $\mathrm{P}(\mathrm{i}, \mathrm{j})$. The process is repeated for pixels in all the neighborhood pixels of the center pixel $\mathrm{P}(\mathrm{i}, \mathrm{j})$ by sliding the $5 \times 5$ window. This way the whole image is enhanced. Finally, after applying these IF-THEN rules, the top and bottom hat filters are applied to the fuzzy-enhanced image in order to further enhance the image.

\section{EXPERIMENTAL RESULTS}

Multiple Iris images from CASIA database were taken for the experiment. For removing noise (salt-pepper) from the Iris images, all the three techniques were applied, and outputs obtained from all the techniques were compared. The PSNR and SSIM values are shown in the table 1, and the quality of the images are measured through it. The comparisons are shown graphically in figure 6 . The images obtained from different techniques are shown in figure 7. The resulting enhanced images obtained from all the three techniques clearly show that the proposed method of image enhancement gives better performance in most of the cases than the rest two techniques.

\section{CONCLUSION}

The proposed algorithm uses the hybrid fuzzy system to enhance the iris image. The proposed system was applied on 40 different types of Iris images to check the level of enhancement. The enhanced images were compared with existing methods and showed the improved quality of the images. Still some more operations like wavelet filtering can be applied to make images appear more robust, further study is being done on fuzzification for better results. To make images appear clearer, some other methods like complex Gabor wavelets of different sizes can also be applied in order to fill the cuts and removing the unnecessary lines that are found in bad-quality iris images.

\section{REFERENCES}

[1] Gonzalez, R.C .and Woods, R.E. 2001. Digital Image Processing, 2nd ed. Englewood Cliffs, NJ: Prentice-Hall.

[2] Bovik, A.C., Huang, T.S. and Munson, D.C. 1983. A generalization of median filtering using linear combinations of order statistics. IEEE Transactions on Acoustics, Speech, and Signal Processing, 31(6) (December 1983),:1342-1349.

[3] Brownrigg, D. 1984. "The weighted median filter," Commun. Assoc. Comput., (Mar. 1984) pp. 807-818.

[4] Ko, S.J. and Lee, S.J. 1991. "Center weighted median filters and their applications to image enhancement," IEEE Trans. Circuits Syst., vol. 15, no. 9 (Sep. 1991), pp. 984-993.

[5] Kerre, E. and Nachtegael, M. Eds. 2000, Fuzzy Techniques in Image Processing. New York: SpringerVerlag, vol. 52, Studies in Fuzziness and Soft Computing.

[6] Thanh, N.M. and Chen, M. "Image Denoising Using Adaptive Neuro-Fuzzy System”, IJAM_36_1_11, Feb. 2007.

[7] Russo, F. and Ramponi, G. "A fuzzy operator for the enhancement of blurred and noisy images," IEEE Trans. Image Processing, vol. 4, pp. 1169-1174, Aug. 1995.

[8] C.-S. Lee, Y.-H. Kuo, and P.-T. Yu, "Weighted fuzzy mean filters for image processing," Fuzzy Sets Syst., no. 89, pp. 157-180, 1997.

[9] Farbiz F. and Menhaj, M.B. 2000. Fuzzy Techniques in Image Processing. New York: Springer-Verlag, vol. 52, Studies in Fuzziness and Soft Computing, ch. A fuzzy logic control based approach for image filtering, pp. 194-221.

[10] Huang, T.S., Yang, G.J. and Tang, G.Y. 1979. "Fast Two-Dimensional Median Filtering Algorithm," IEEE Transactions on Acoustics, Speech, and Signal Processing, 1, pp. 13-18.

[11] Nodes, T.A. and Gallagher, N.C. Jr. 1984. "The Output Distribution of Median Type Filters," IEEE Transactions on Communications, COM-32. 
[12] Hwang, H. and Haddad, R.A. 1995. "Adaptive median filters: new algorithms and results," IEEE Transactions on Image Processing, 4, pp. 499-502.

[13] Chen, T. and Wu, H.R. 2001. "Space variant median filters for the restoration of impulse noise corrupted images," IEEE Transactions on Circuits and Systems II, 48, pp. 784-789.

[14] H.-L. Eng and K.-K. Ma, "Noise Adaptive SoftSwitching Median Filter," IEEE Transactions on Image Processing, 10, pp. 242-251,2001.

[15] G. Pok, J.C. Liu, and A. S. Nair, "Selective Removal of Impulse Noise Based on Lomogeneity level Information," IEEE Transactions on Image Processing, 12 , pp. 85-92,2003.

[16] Mamdani, E.H. and Assilian, S. 1975. "An experiment in linguistic synthesis with a fuzzy logic controller," International Journal of Man-Machine Studies, 7(1):1-13.
[17] Takagi,T. and Sugeno, M. "Fuzzy identification of systems and its applications to modeling and control," IEEE Trans. Syst., Man, Cybern., vol. 15, pp. 116-132, Jan. 1985.

[18] Buckley, J.J. and Feuring, T. 1999. Fuzzy and Neural: Interactions and Applications, ser. Studies in Fuzziness and Soft Computing. Heidelberg, Germany: PhysicaVerlag.

[19] Lin, C.T. and C. S. George Lee, C.S. 1996. Neural Fuzzy Systems--ANeuro-Fuzzy Synergism to Intelligent Systems. Englewood Cliffs, NJ:Prentice-Hall.

[20] J. S. R. Jang, C. T. Sun, and E. Mizutani, 1997. NeuroFuzzy and Soft Computing. Englewood Cliffs, NJ: Prentice-Hall.

Table 1. Comparison of wiener, median and proposed enhancement methods based on PSNR and SSIM

\begin{tabular}{|c|c|c|c|c|c|c|}
\hline Image & \multicolumn{2}{|c|}{ Wiener Filter } & \multicolumn{2}{|c|}{ Median Filter } & \multicolumn{2}{c|}{ Proposed method(IEHFS) } \\
\hline Iris Image & PSNR & SSIM & PSNR & SSIM & PSNR & SSIM \\
\hline 001_1_1 & 56.4991 & 0.98283 & 56.5485 & 0.98186 & 56.5932 & 0.98277 \\
\hline 001_1_2 & 56.3661 & 0.98269 & 56.4001 & 0.98118 & 56.461 & 0.98185 \\
\hline 001_2_1 & 56.6289 & 0.98294 & 56.7868 & 0.98324 & 56.8557 & 0.98347 \\
\hline 001_2_2 & 56.5216 & 0.9823 & 56.6941 & 0.98327 & 56.7344 & 0.98321 \\
\hline 002_1_1 & 56.5037 & 0.98192 & 57.9033 & 0.98751 & 57.9282 & 0.98744 \\
\hline 002_1_2 & 56.5009 & 0.98253 & 57.4439 & 0.98605 & 57.5148 & 0.98605 \\
\hline 002_2_1 & 56.7142 & 0.98206 & 57.7897 & 0.98708 & 57.8148 & 0.98698 \\
\hline 002_2_2 & 56.4521 & 0.98279 & 57.3151 & 0.98557 & 57.387 & 0.9858 \\
\hline 003_1_1 & 56.651 & 0.98489 & 56.8028 & 0.98354 & 56.8201 & 0.98359 \\
\hline 003_1_2 & 56.7778 & 0.98498 & 56.7357 & 0.98322 & 56.7625 & 0.98331 \\
\hline 003_2_1 & 56.7367 & 0.9842 & 57.1149 & 0.9846 & 57.1156 & 0.98447 \\
\hline 003_2_2 & 56.749 & 0.98472 & 56.8904 & 0.9843 & 56.9993 & 0.98453 \\
\hline 004_1_1 & 57.5354 & 0.98577 & 58.0547 & 0.98671 & 58.0744 & 0.98671 \\
\hline 004_1_2 & 57.5195 & 0.98567 & 57.7743 & 0.98615 & 57.8091 & 0.98612 \\
\hline 004_2_1 & 57.5127 & 0.98567 & 57.9376 & 0.98653 & 57.9704 & 0.98655 \\
\hline 004_2_2 & 57.4979 & 0.98588 & 57.7563 & 0.98609 & 57.8238 & 0.98622 \\
\hline 008_1_1 & 56.577 & 0.98191 & 57.7671 & 0.98648 & 57.800 & 0.98675 \\
\hline 008_1_2 & 56.4692 & 0.98122 & 57.8725 & 0.98666 & 57.9042 & 0.98701 \\
\hline 008_2_1 & 56.7276 & 0.98337 & 57.0463 & 0.98392 & 57.0727 & 0.98411 \\
\hline 008_2_2 & 56.8442 & 0.98322 & 57.8588 & 0.98658 & 57.9008 & 0.98689 \\
\hline 009_1_1 & 56.6764 & 0.98214 & 57.2764 & 0.98456 & 57.3267 & 0.98482 \\
\hline 009_1_2 & 56.9251 & 0.98298 & 57.904 & 0.98325 & 56.9967 & 0.98396 \\
\hline 009_2_1 & 56.8162 & 0.98307 & 56.7731 & 0.9823 & 56.8891 & 0.98343 \\
\hline 009_2_2 & 56.9126 & 0.98232 & 56.3946 & 0.9849 & 57.4498 & 0.98531 \\
\hline 010_1_1 & 57.0617 & 0.98469 & 57.599 & 0.98643 & 57.6149 & 0.98626 \\
\hline 010_1_2 & 56.7501 & 0.98357 & 57.7882 & 0.98683 & 57.7583 & 0.98649 \\
\hline 010_2_1 & 56.7589 & 0.98379 & 57.1073 & 0.98471 & 57.1464 & 0.98472 \\
\hline 010_2_2 & 56.4447 & 0.98302 & 57.453 & 0.98641 & 57.4581 & 0.98615 \\
\hline
\end{tabular}




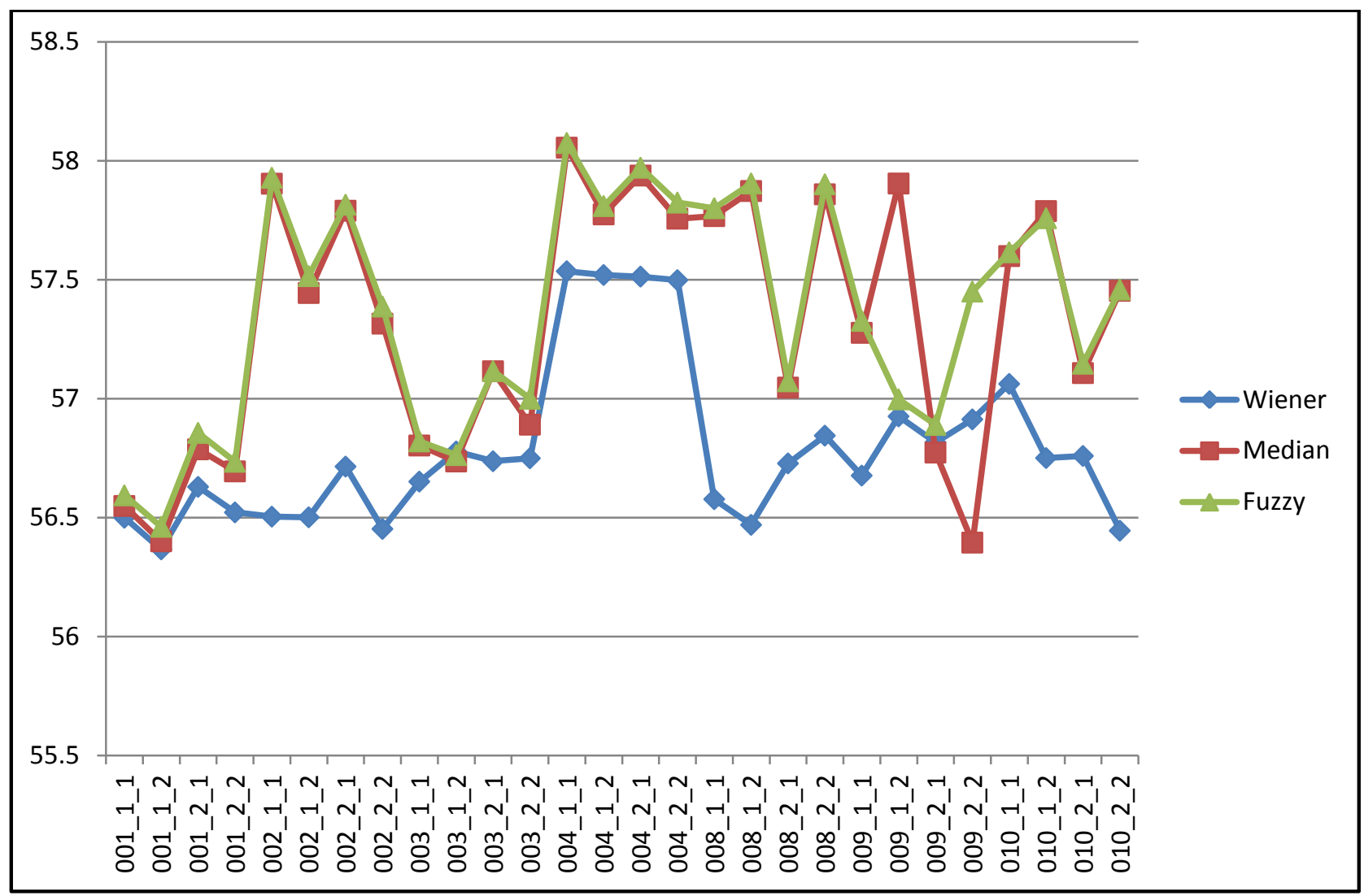

Fig 6: Graphical representation of PSNR and SSIM of Wiener, median and Proposed enhancement methods) 
(a)

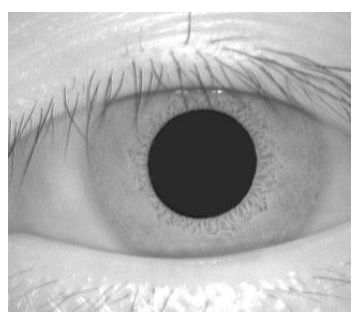

(b)

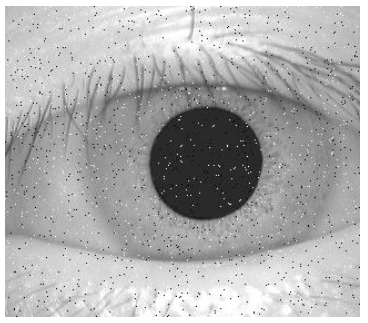

(c)

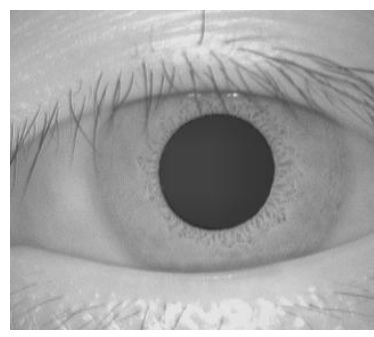

(d)

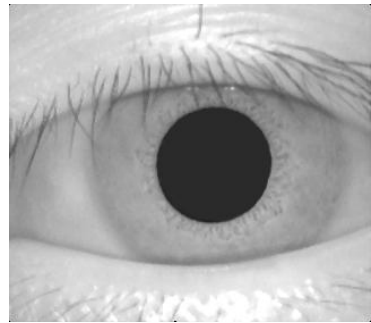

(e)

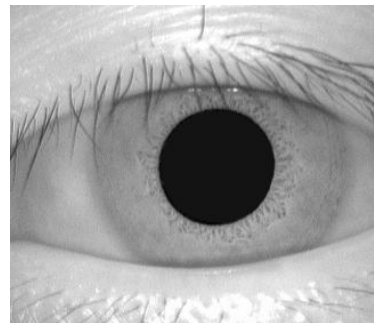

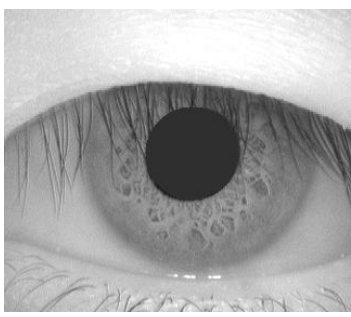
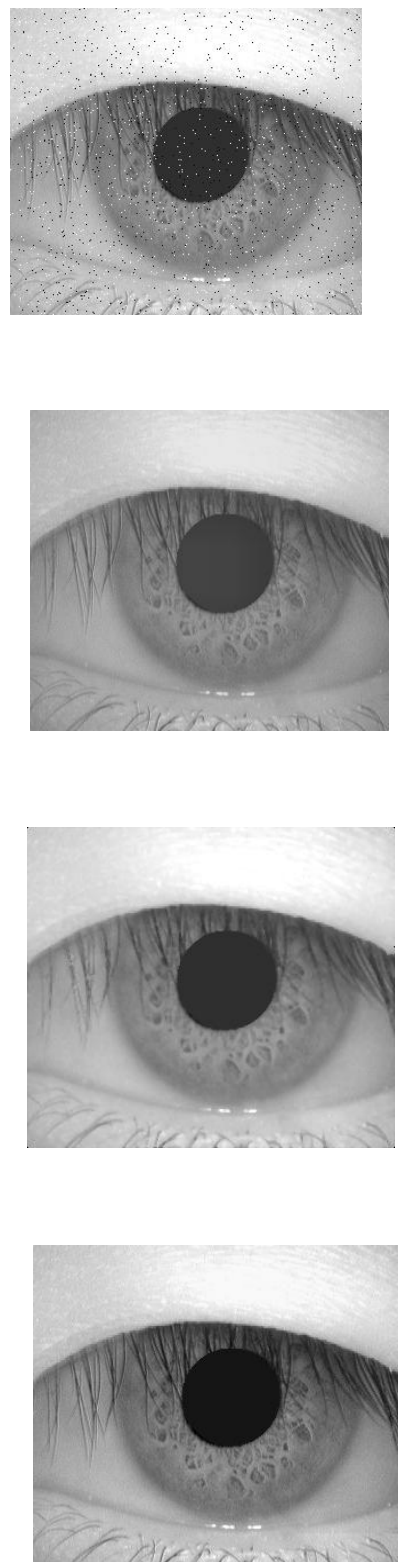
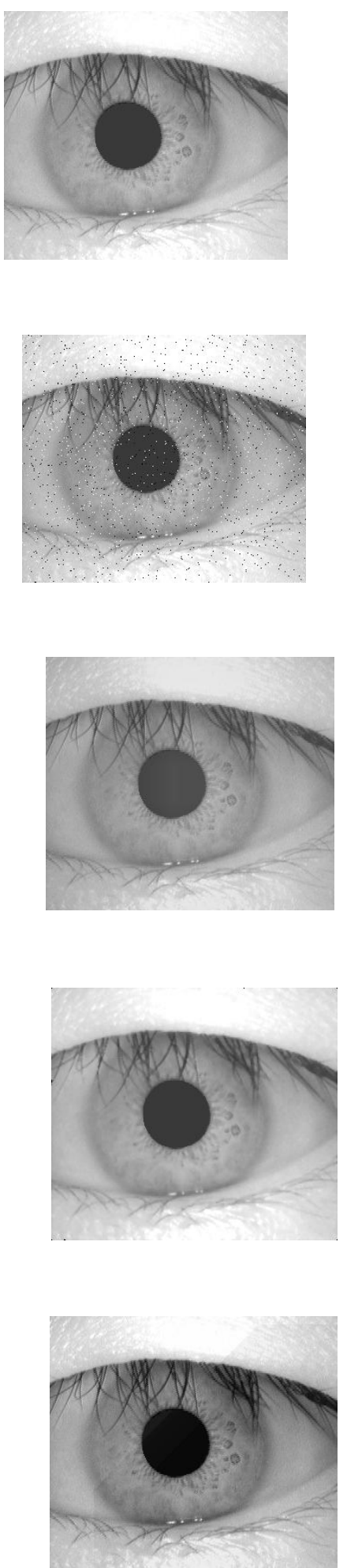

Fig 7: Enhancement of iris image a) Original image b) Noisy image c) Wiener-filtered image d) Median-filtered image e) Enhancement using proposed method (IEHFS) 